\title{
Some Identities Related with the Higher-order Deformed Degenerate Bernoulli and Euler Polynomials
}

\author{
Lee Chae Jang \\ Graduate School of Education, Konkuk University, Seoul, Republic of Korea
}

Email address:

lcjang@konkuk.ac.kr

\section{To cite this article:}

Lee Chae Jang. Some Identities Related with the Higher-order Deformed Degenerate Bernoulli and Euler Polynomials. Applied and Computational Mathematics. Vol. 6, No. 6, 2017, pp. 254-258. doi: 10.11648/j.acm.20170606.13

Received: July 25, 2017; Accepted: November 10, 2017; Published: December 15, 2017

Abstract: Recently, Kim-Kim (2016-2017) studied simmetric identities of higher-order degenerate Bernoulli and Euler polynomials which were defined by Carlitz (1979). In this paper, we define the higher-order deformed degenerate Bernoulli and Euler polynomials which are modified the higher-order degenerate Bernoulli and Euler polynomials. We also investigate some interesting identities for the the higher-order deformed degenerate Bernoulli and Euler polynomials.

Keywords: Bernoulli Polynomials, Euler Polynomials, Degenerate Bernoulli Polynomials

\section{Introduction}

Let $p$ be a odd prime number with of $p \equiv 1(\bmod 1)$. Throughout this paper, $Z_{p}, Q_{p}$, and $C_{p}$ denote the ring of $p$-adic integers, the filed of $p$-adic rational numbers, and the completion of the algebraic closure of $Q_{p}$, respectively. The $p$-adic norm $|\cdot|_{p}$ is normalized as $|p|_{p}=\frac{1}{p}$. Let $U D\left(Z_{p}\right)$ be the space of uniformly differentiable functions on $Z_{p}$. For $f \in U D\left(Z_{p}\right)$, the fermionic $p$-adic integrals on $Z_{p}$ is defined by Kim to be

$$
I_{-1}(f)=\int_{\mathbb{Z}_{p}} f(x) d \mu_{-1}(x)=\lim _{N \rightarrow \infty} \sum_{x=0}^{p^{N}-1} f(x)(-1)^{x},
$$

(see $[6-10,14,17,23,25,28,34,38,39])$. From (1), it is well-known that

$$
I_{-1}\left(f_{1}\right)+I_{-1}(f)=2 f(0),
$$

where $f_{1}(x)=f(x+1)$. The bosonic integral on $\mathbb{Z}_{p}$ is defined by Volkenborn as

$$
I_{0}(f)=\int_{Z_{p}} f(x) d \mu_{0}(x)=\lim _{N \rightarrow \infty} \frac{1}{p^{N}} \sum_{x=0}^{p^{N}-1} f(x),
$$

(see $[1,11,12,13,15,20,22,24,26,27,33,35,36])$. Then, by (3), it is well-known that

$$
I_{0}\left(f_{1}\right)-I_{0}(f)=f^{\prime}(0),
$$

where $f_{1}(x)=f(x+1)$ and $f^{\prime}(x)=\left.\frac{d f(x)}{d x}\right|_{x=0}$. For $r \in \mathbb{N}$, we consider the higher-order Bernoulli polynomials which are given by the generating function as

$$
\left(\frac{t}{e^{t}-1}\right)^{r} e^{x t}=\sum_{n=0}^{\infty} B_{n}^{(r)}(x) \frac{t^{n}}{n !}
$$

When $x=0, B_{n}^{(r)}=B_{n}^{(r)}(0)$ are called the higher-order Bernoulli numbers. For $r \in \mathbb{N}$, we consider the higher-order Euler polynomials which are given by the generating function as

$$
\left(\frac{2}{e^{t}+1}\right)^{r} e^{x t}=\sum_{n=0}^{\infty} E_{n}^{(r)}(x) \frac{t^{n}}{n !} .
$$

When $x=0, E_{n}^{(r)}=E_{n}^{(r)}(0)$ are called the higher-order Euler numbers (see $[8,16,19,21,29])$. Note that $B_{n}(x)=$ $B_{n}^{(1)}(x)$ and $E_{n}(x)=E_{n}^{(1)}(0)$ are the Bernoulli polynomials and the Euler polynomials, respectively. In $[1,4,5,18,30,31$, $32,37]$, we recall that the Stirling numbers of the second kind are given by

$$
x^{n}=\sum_{l=0}^{n} S_{2}(n, l)(x)_{l},(n \geq 0),
$$

and

$$
\left(e^{t}-1\right)^{n}=n ! \sum_{l=n}^{\infty} S_{2}(n, m) \frac{t^{l}}{l !},(n \geq 0)
$$

and the Stirling numbers of the first kind are given by 


$$
(x)_{n}=\sum_{l=0}^{n} S_{1}(n, l) x^{l},(n \geq 0),
$$

and

$$
(\log (1+t))^{n}=n ! \sum_{l=n}^{\infty} S_{1}(n, l) \frac{t^{l}}{l !},(n \geq 0)
$$

For $\lambda \neq 0$, the degenerate Bernoulli polynomials are defined by Carlitz as the generating function to be

$$
\frac{t}{(1+\lambda t)^{\frac{1}{\lambda}}-1}(1+\lambda t)^{\frac{x}{\lambda}}=\sum_{n=0}^{\infty} \beta_{n}(\lambda, x) \frac{t^{n}}{n !},
$$

(see $[2,3,11,13,15,22,24,27,33,35,36])$. When $x=0$, $\beta_{n}(\lambda)=\beta_{n}(\lambda, 0)$ are called the degenerate Bernoulli polynomials.

Note that $\quad \lim _{\lambda \rightarrow 0} \beta_{n}(\lambda, \lambda x)=B_{n}(x) \quad$ and $\lim _{\lambda \rightarrow \infty} \lambda^{-n} \beta_{n}(\lambda, \lambda x)=b_{n}(x)$, where $b_{n}(x)$ are the Bernoulli polynomials of the second kind given by the generating function

$\frac{t}{\log (1+t)}(1+t)^{x}=\sum_{n=0}^{\infty} b_{n}(x) \frac{t^{n}}{n !},(\operatorname{see}[20,32,35,37])$.

From (8), we observe that

$$
\begin{gathered}
\frac{t}{\log (1+t)}(1+t)^{x}=\frac{t}{\log (1+t)} e^{x \log (1+t)}= \\
\sum_{n=0}^{\infty}\left(b_{m} \frac{t^{m}}{m !} \sum_{l}^{\infty} \sum_{k=0}^{l} x^{k} S_{1}(l, k)\right) \frac{t^{l}}{l !}
\end{gathered}
$$

From (8) and (9), we obtain the following

$$
b_{m}=\sum_{l=0}^{n} \sum_{k=0}^{l}\left(\begin{array}{l}
n \\
l
\end{array}\right) b_{n-l} x^{k} S_{1}(l, k)
$$

The degenerate Euler polynomials are defined by Carlitz as the generating function to be

$$
\frac{2}{(1+\lambda t)^{\frac{1}{\lambda}}+1}(1+\lambda t)^{\frac{x}{\lambda}}=\sum_{n=0}^{\infty} \mathcal{E}(\lambda, x) \frac{t^{n}}{n !}
$$

When $x=0, \varepsilon_{n}(\lambda)=\varepsilon_{n}(\lambda, 0)$ are called the degenerate Euler numbers. Note that $\lim _{\lambda \rightarrow 0} \varepsilon_{n}(\lambda, x)=\mathcal{E}(x)$. For $r \in \mathbb{N}$, Carlitz [1, 2] studied the higher-order degenerate Bernoulli polynomials and the higher-order degenerate Euler polynomials which are defined by the generating functions, respectively

$$
\left(\frac{t}{(1+\lambda t)^{\frac{1}{\lambda}}-1}\right)^{r}(1+\lambda t)^{\frac{x}{\lambda}}=\sum_{n=0}^{\infty} \beta_{n}^{(r)}(\lambda, x) \frac{t^{n}}{n !},
$$

and

$$
\left(\frac{2}{(1+\lambda t)^{\frac{1}{\lambda}}+1}\right)^{r}(1+\lambda t)^{\frac{x}{\lambda}}=\sum_{n=0}^{\infty} \mathcal{E}^{(r)}(\lambda, x) \frac{t^{n}}{n !} .
$$

When $x=0, \beta_{n}^{(r)}(\lambda)=\beta_{n}^{(r)}(\lambda, 0)$ and $\mathcal{E}^{(r)}(\lambda)=$ $\mathcal{E}_{n}(\lambda, 0)$ are called the higher-order degenerate Bernoulli numbers and the higher-order degenerate Euler numbers, respectively. Note that $\beta_{n}^{(1)}(\lambda, x)=\beta_{n}(\lambda, x)$ and $\mathcal{E}_{n}^{(1)}(\lambda, x)=\varepsilon_{n}(\lambda, x)$. From (16), we observe that for $r \in \mathbb{N}$,

$$
\begin{gathered}
\sum_{n=0}^{\infty} \beta_{n}^{(r)}(\lambda, x) \frac{t^{n}}{n !}=\left(\frac{t}{(1+\lambda t)^{\frac{1}{\lambda}}-1}\right)^{r}(1+\lambda t)^{\frac{x}{\lambda}}= \\
\left(\frac{\lambda t}{\log (1+\lambda t)}\right)^{r}\left(\frac{\frac{1}{\lambda} \log (1+\lambda t)}{e^{\frac{1}{\lambda} \log (1+\lambda t)}-1}\right)^{r} e^{\frac{x}{\lambda} \log (1+\lambda t)} \\
=\sum_{n=0}^{\infty}\left(\sum_{m=0}^{n} \sum_{k=0}^{m}\left(\begin{array}{l}
n \\
m
\end{array}\right) b_{n-m}^{(r)} \lambda^{n-k} B_{k}^{(r)} S_{1}(m, k)\right) \frac{t^{n}}{n !}
\end{gathered}
$$

where $b_{n}$ are the higher-order Bernoulli numbers of second kind which are given by the generating functions to be

$$
\left(\frac{t}{\log (1+t)}\right)^{r}=\sum_{n=0}^{\infty} b_{n}^{(r)} \frac{t^{n}}{n !}
$$

From (13), we obtain the following identity

$$
\beta_{n}^{(r)}(\lambda, x)=\sum_{m=0}^{n} \sum_{k=0}^{m}\left(\begin{array}{l}
n \\
m
\end{array}\right) b_{n-m}^{(r)} \lambda n-k B_{k}^{(r)} S_{1}(m, k) .
$$

From (17), we also observe that

$$
\begin{gathered}
\sum_{n=0}^{\infty} \varepsilon_{n}^{(r)}(\lambda, x) \frac{t^{n}}{n !}=\left(\frac{2}{(1+\lambda t)^{\frac{1}{\lambda}}+1}\right)^{r}(1+\lambda t)^{\frac{x}{\lambda}}= \\
\sum_{n=0}^{\infty}\left(\sum_{l=0}^{n} E_{l}^{(r)}(x) S_{1}(n, l) \lambda^{n-l}\right) \frac{t^{n}}{n !} .
\end{gathered}
$$

From (21), we obtain the following identity.

$$
\mathcal{E}_{n}^{(r)}(\lambda, x)=\sum_{l=l}^{n} E_{l}^{(r)}(x) S_{1}(n, l) \lambda^{n-l} .
$$

In this paper, we consider the higher-order degenerate Bernoulli and Euler polynomials which were defined by Carlitz and define the higher-order deformed degenerate Bernoulli and Euler polynomials which are modified the higher-order degenerate Bernoulli and Euler polynomials. We also investigate some interesting identities for the the higher-order deformed degenerate Bernoulli and Euler polynomials

\section{The Higher-Order Deformed Degenerate Bernoulli and Euler Polynomials}

For $\lambda, t \in \mathbb{C}_{p}$ with $|t|_{p}<p^{-\frac{1}{p-1}}$ and $|\lambda|_{p}<p^{-\frac{1}{p-1}}$, we define the deformed degenerate Bernoulli polynomials which are given by the generating functions to be

$$
\frac{t}{(1+\lambda)^{\frac{t}{\lambda}}-1}(1+\lambda)^{\frac{x t}{\lambda}}=\sum_{n=0}^{\infty} \hat{\beta}_{n}(\lambda, x) \frac{t^{n}}{n !} .
$$

and the deformed degenerate Euler polynomials which are given by the generating functions to be

$$
\frac{2}{(1+\lambda)^{\frac{t}{\lambda}+1}}(1+\lambda)^{\frac{x t}{\lambda}}=\sum_{n=0}^{\infty} \hat{\varepsilon}_{n}(\lambda, x) \frac{t^{n}}{n !} .
$$

When $x=0, \hat{\beta}_{n}(\lambda)=\hat{\beta}_{n}(\lambda, 0)$ and $\hat{\varepsilon}_{n}(\lambda)=\hat{\varepsilon}_{n}(\lambda, 0)$ are called the deformed degenerate Euler numbers. We note that 


$$
\lim _{\lambda \rightarrow 0} \frac{t}{(1+\lambda)^{\frac{t}{\lambda}}-1}(1+\lambda)^{\frac{t x}{\lambda}}=\frac{t}{e^{t}-1} e^{t x}=\sum_{n=0}^{\infty} B_{n}(x) \frac{t^{n}}{n !}
$$

and

$$
\lim _{\lambda \rightarrow 0} \frac{2}{(1+\lambda)^{\frac{t}{\lambda}}+1}(1+\lambda)^{\frac{t x}{\lambda}}=\frac{2}{e^{t}+1} e^{t x}=\sum_{n=0}^{\infty} E_{n}(x) \frac{t^{n}}{n !} .
$$

From (25) and (26), we see that $\lim _{\lambda \rightarrow 0} \hat{\beta}_{n}(\lambda, x)=B_{n}(x)$ and $\lim _{\lambda \rightarrow 0} \hat{E}_{n}(\lambda, x)=E_{n}(x)$. If we take $f(y)=(1+$ $\lambda)^{\frac{(x+y) t}{\lambda}}$, by (4) and (23), we get

$$
\left(\frac{\lambda}{\log (1+\lambda)}\right) \int_{\mathbb{Z}_{p}}(1+\lambda)^{\frac{(x+y) t}{\lambda}} d \mu_{0}(y)=\sum_{n=0}^{\infty} \hat{\beta}_{n}(\lambda, x) \frac{t^{n}}{n !} .
$$

By (2) and (24), we have

$$
\begin{gathered}
\int_{\mathbb{Z}_{p}}(1+\lambda)^{\frac{(x+y) t}{\lambda}} d \mu_{-1}(y)=\frac{2}{(1+\lambda)^{\frac{t}{\lambda}}+1}(1+\lambda)^{\frac{t x}{\lambda}}= \\
\sum_{n=0}^{\infty} \hat{\varepsilon}_{n}(\lambda, x) \frac{t^{n}}{n !} .
\end{gathered}
$$

For $r \in \mathbb{N}$, the higher-order deformed degenerate Bernoulli polynomials are defined by the generating functions to be

$$
\left(\frac{t}{(1+\lambda)^{\frac{t}{\lambda}}-1}\right)^{r}(1+\lambda)^{\frac{x t}{\lambda}}=\sum_{n=0}^{\infty} \hat{\beta}_{n}^{(r)}(\lambda, x) \frac{t^{n}}{n !}
$$

and the higher-order deformed degenerate Euler polynomials are defined by the generating functions to be

$$
\left(\frac{2}{(1+\lambda)^{\frac{t}{\lambda}}+1}\right)^{r}(1+\lambda)^{\frac{x t}{\lambda}}=\sum_{n=0}^{\infty} \hat{\mathcal{E}}_{n}^{(r)}(\lambda, x) \frac{t^{n}}{n !}
$$

$$
\int_{\mathbb{Z}_{p}} \cdots \int_{\mathbb{Z}_{p}}(1+\lambda)^{\frac{\left(x+x_{1}+\cdots+x_{r}\right) t}{\lambda}} d \mu_{-1}\left(x_{1}\right) \cdots d \mu_{-1}\left(x_{r}\right)=\sum_{l=0}^{\infty} \hat{\varepsilon}_{l}^{(r)}(\lambda, x) \frac{t^{l}}{l !}
$$

From (34) and (35), we obtain the following identity.

Theorem 2.2 For $n \geq 0$, we have

$$
\hat{\mathcal{E}}_{l}^{(r)}(\lambda, x)=\sum_{n=l}^{\infty} S_{1}(n, l) \lambda^{n-l} E_{l}^{(r)}(x) \frac{l !}{n !} .
$$

Now we investigate the following distribution relation on the higher-order deformed degenerate Bernoulli polynomials. For $d \in \mathbb{N}$, we observe that

$$
t \sum_{a=0}^{d-1}(1+\lambda)^{\frac{a t}{\lambda}}=\frac{t\left((1+\lambda)^{\frac{d t}{\lambda}}-1\right)}{(1+\lambda)^{\frac{t}{\lambda}}-1}
$$

and hence

$\frac{t}{(1+\lambda)^{\frac{d t}{\lambda}}-1} \sum_{a=0}^{d-1}(1+\lambda)^{\frac{a t}{\lambda}}(1+\lambda)^{\frac{x t}{\lambda}}=\frac{t}{(1+\lambda)^{\frac{t}{\lambda}}-1}(1+\lambda)^{\frac{x t}{\lambda}}$

Thus, by (37) and (38), we have

$$
\frac{t}{(1+\lambda)^{\frac{t}{\lambda}}-1}(1+\lambda)^{\frac{x t}{\lambda}}=\sum_{n=0}^{\infty} d^{n-1} \sum_{a=0}^{d-1} \hat{\beta}_{n}\left(\lambda, \frac{a+x}{d}\right) \frac{t^{n}}{n !}
$$

From (23) and (39), we obtain the following identity. Theorem 2.3 For $n \geq 0$ and $d \in \mathbb{N}$, we have
When $x=0, \hat{\beta}_{n}^{(r)}(\lambda)=\hat{\beta}_{n}^{(r)}(\lambda, 0)$ and $\hat{\mathcal{E}}_{n}^{(r)}(\lambda)=$ $\hat{\mathcal{E}}_{n}^{(r)}(\lambda, 0)$ are called the higher-order deformed degenerate Bernoulli numbers and the higher-order deformed degenerate Euler numbers, respectively. We observe that

$$
\begin{aligned}
\int_{\mathbb{Z}_{p}} \cdots & \int_{\mathbb{Z}_{p}}(1+\lambda)^{\frac{\left(x+x_{1}+\cdots+x_{r}\right) t}{\lambda}} d \mu_{0}\left(x_{1}\right) \cdots d \mu_{0}\left(x_{r}\right)= \\
& \sum_{l=0}^{\infty} \sum_{n=l}^{\infty} S_{1}(n, l) \lambda^{n-l} B_{l}^{(r)}(x) \frac{l !}{n !} \frac{t^{l}}{l !}
\end{aligned}
$$

and

$$
\begin{array}{r}
\left(\frac{\lambda}{\log (1+\lambda)}\right)^{r} \int_{\mathbb{Z}_{p}} \cdots \int_{\mathbb{Z}_{p}} \\
\lambda)^{\frac{\left(x+x_{1}+\cdots+x_{r}\right) t}{\lambda}} d \mu_{0}\left(x_{1}\right) \cdots d \mu_{0}\left(x_{r}\right)=\sum_{l=0}^{\infty} \hat{\beta}_{l}^{(r)}(\lambda, x) \frac{t^{l}}{l !}
\end{array}
$$

From (31) and (32), we obtain the following identity. Theorem 2.1 For $n \geq 0$, we have

$\hat{\beta}_{l}^{(r)}(\lambda, x)=\left(\frac{\lambda}{\log (1+\lambda)}\right)^{r} \sum_{n=l}^{\infty} S_{1}(n, l) \lambda^{n-l} B_{l}^{(r)}(x) \frac{l !}{n !}$.

We also observe that

$$
\begin{gathered}
\int_{\mathbb{Z}_{p}} \cdots \int_{\mathbb{Z}_{p}}(1+\lambda)^{\frac{\left(x+x_{1}+\cdots+x_{r}\right) t}{\lambda}} d \mu_{-1}\left(x_{1}\right) \cdots d \mu_{-1}\left(x_{r}\right) \\
=\sum_{l=0}^{\infty} \sum_{n=l}^{\infty} S_{1}(n, l) \lambda^{n-l} E_{l}^{(r)}(x) \frac{l !}{n !} \frac{t^{l}}{l !}
\end{gathered}
$$

and

$$
\hat{\beta}_{n}(\lambda, x)=d^{n-1} \sum_{a=0}^{d-1} \hat{\beta}_{n}\left(\lambda, \frac{a+x}{d}\right) .
$$

For $r \in \mathbb{N}$, we also observe that

$$
\begin{array}{r}
\left(\frac{t}{(1+\lambda)^{\frac{t}{\lambda}}-1}\right)^{r}(1+\lambda)^{\frac{x t}{\lambda}}= \\
\sum_{n=0}^{\infty}\left(d^{n-r} \sum_{a_{1}, \cdots, a_{r}=0}^{d-1} \hat{\beta}_{n}\left(\lambda, \frac{a_{1}+\cdots+a_{r}+x}{d}\right)\right) \frac{t^{n}}{n !} .
\end{array}
$$

From (29) and (41), we obtain the following identity.

Theorem 2.4 For $n \geq 0$ and $d, r \in \mathbb{N}$, we have

$$
\hat{\beta}_{n}^{(r)}(\lambda, x)=d^{n-r} \sum_{a_{1}, \cdots, a_{r}=0}^{d-1} \hat{\beta}_{n}^{(r)}\left(\lambda, \frac{a_{1}+\cdots+a_{r}+x}{d}\right) .
$$

We also investigate the following distribution relation on the higher-order deformed degenerate Euler polynomials. For an odd positive integer $d$, we observe that

$$
\begin{array}{r}
\int_{\mathbb{Z}_{p}} \cdots \int_{\mathbb{Z}_{p}}(1+\lambda)^{\frac{\left(x+x_{1}+\cdots+x_{r}\right) t}{\lambda}} d \mu_{-1}\left(x_{1}\right) \cdots d \mu_{-1}\left(x_{r}\right) \\
=\sum_{n=0}^{\infty} \sum_{a_{1}, \cdots, a_{r}=0}^{d-1}(-1)^{a_{1}+\cdots+a_{r}} \hat{\mathcal{E}}_{n}^{(r)}\left(\lambda, \frac{x+a_{1}+\cdots+a_{r}}{d}\right) \frac{t^{n}}{n !}
\end{array}
$$


From (30)and (43), we obtain the following identity.

Theorem 2.5 For $n \geq 0, r \in \mathbb{N}$ and $d \equiv 1(\bmod 2)$, we have

$$
\hat{\mathcal{E}}_{n}^{(r)}(\lambda, x)=\sum_{a_{1}, \cdots, a_{r}=0}^{d-1}(-1)^{a_{1}+\cdots+a_{r}} \hat{\mathcal{E}}_{n}^{(r)}\left(\lambda, \frac{x+a_{1}+\cdots+a_{r}}{d}\right) .
$$

Finally, by replacing $t$ by $\frac{\lambda t}{\log (1+\lambda)}$ in (29) and (30), we derive that

$$
\begin{array}{r}
\sum_{n=0}^{\infty} \hat{\beta}_{n}(\lambda, x)\left(\frac{\lambda}{\log (1+\lambda)}\right)^{r} \frac{t^{n}}{n !}= \\
\sum_{n=0}^{\infty}\left(\left(\frac{\lambda}{\log (1+\lambda)}\right)^{r} \sum_{l=0}^{n} B_{l}^{(r)}\left(\begin{array}{l}
n \\
l
\end{array}\right) x^{n-l}\right) \frac{t^{n}}{n !}, \quad(45)
\end{array}
$$

and

$$
\sum_{n=0}^{\infty} \hat{\varepsilon}_{n}(\lambda, x)\left(\frac{\lambda t}{\log (1+\lambda)}\right)^{n} \frac{1}{n !}=\sum_{n=0}^{\infty}\left(\sum_{l=0}^{n}\left(\begin{array}{l}
n \\
l
\end{array}\right) E_{l}^{(r)} x^{n-l}\right) \frac{t^{n}}{n !} .
$$

Thus by comparing the coefficients of (45) and(46), we obtain the following identities.

Theorem 2.6 For $n \geq 0$ and $r \in \mathbb{N}$, we have

$$
\hat{\beta}_{n}^{(r)}(\lambda, x)=\sum_{l=0}^{n}\left(\begin{array}{l}
n \\
l
\end{array}\right) B_{l}^{(r)} x^{n-l} .
$$

and

$$
\hat{\mathcal{E}}_{n}^{(r)}(\lambda, x)=\left(\frac{\log (1+\lambda)}{\lambda}\right)^{r} \sum_{l=0}^{n}\left(\begin{array}{l}
n \\
l
\end{array}\right) E_{l}^{(r)} x^{n-l} .
$$

\section{Conclusions}

This study was to define the deformed degenerate Bernoulli and Euler polynomials in (23), (24) and the higher-order deformed degenerate Bernoulli and Euler polynomials in (29), (30). We obtained useful identities for the higher-order deformed degenerate Bernoulli and Euler polynomials in Theorem 2.1, 2.2, 2.3, 2.4, 2.5, and 2.6.

We suggest to find the differential equations whose solution is the generating function of the higher-order deformed degenerate Bernoulli and Euler polinomials, and to investigate the symmetric identities of the higher-order deformed degenerate Bernoulli and Euler polynomials.

\section{References}

[1] L. Carlitz, Degenerate Stirling, Bernoulli and Eulerian numbers, Utilitas Math., 15(1979), no.2, 51-88.

[2] L. Carlitz, A degenerate Staudt-Clausen theorem, Arch. Math. (Basel), 7 (1956), 28-33.

[3] T. Kim, D. S. Kim, Degenerate Laplace transform and degenerate gamma function, Russ. J. Math. Phys., 24(2) (2017), 241-248.

[4] D. S. Kim, T. Kim, On degenerate Bell numbers and polynomials, Rev. R. Acad. Cienc. Exactas Fis. Nat. Ser. A Math. RACSAM, 111(2) (2017), 435-446.

[5] D. S. Kim, T. Kim, Some identities of degenerate Daehee numbers arising from certain differential equations, J. Nonlinear Sci. Appl., 10(2) (2017), 744-751.
[6] T. Kim, V. D. Dolgy, H. I. Kwon, Expansions of degenerate q -Euler numbers and polynomials, Proc. Jangjeon Math. Soc., 19(4) (2016), 625-630.

[7] V. D. Dolgy, T. Kim, H. I. Kwon, J. J. Seo, Some identities for degenerate Euler numbers and polynomials arising from degenerate Bell polynomials, Proc. Jangjeon Math. Soc., 19(3) (2016), 457-464.

[8] T. Kim, D. S. Kim, Identities of symmetry for the generalized degenerate Euler polynomials, Proc. Math. Stat., 171 (2016), 35-43.

[9] T. Kim, D. V. Dolgy, L. C. Jang, H. I. Kwon, Some identities of degenerate q-Euler polynomials under the symmetry group of degree n, J. Nonlinear Sci. Appl., 9(6) (2016), 4707-4712.

[10] T. Kim, D. S. Kim, H. I. Kwon, J. J. Seo, Some identities for degenerate Frobenius-Euler numbers arising from nonlinear differential equations, Ital. J. Pure Appl. Math., 36 (2016), 843-850.

[11] T. Kim, On degenerate q -Bernoulli polynomials, Bull. Korean Math. Soc., 53(4) (2016), 1149-1156.

[12] T. Kim, D. S. Kim, J. J. Seo, Fully degenerate poly-Bernoulli numbers and polynomials, Open Math., 14 (2016), 545-556.

[13] D. V. Dolgy, T. Kim, J. J. Seo, On the symmetric identities of modified degenerate Bernoulli polynomials, Proc. Jangjeon Math. Soc., 19(2) (2016), 301-308.

[14] H. I. Kwon, T. Kim, J. J. Seo, Some new identities of symmetry for modified degenerate Euler polynomials, Proc. Jangjeon Math. Soc., 19(2) (2016), 237-242.

[15] T. Kim, D. S. Kim, H. I. Kwon, Some identities relating to degenerate Bernoulli polynomials, Filomat, 30(4) (2016), 905-912.

[16] T. Kim, D. S. Kim, H. I. Kwon, J. J. Seo, Differential equations arising from the generating function of general modified degenerate Euler numbers, Adv. Difference Equ., 2016:129 (2016), 7 pp.

[17] D. S. Kim, T. Kim, D. V. Dolgy, On Carlitz's degenerate Euler numbers and polynomials, J. Comput. Anal. Appl., 21(4) (2016), 738-742.

[18] T. Kim, H. I. Kwon, J. J. Seo, Degenerate q -Changhee polynomials, J. Nonlinear Sci. Appl., 9(5) (2016), 2389-2393.

[19] T. Kim, D. S. Kim, Identities involving degenerate Euler numbers and polynomials arising from non-linear differential equations, J. Nonlinear Sci. Appl., 9(5) (2016), 2086-2098.

[20] D. V. Dolgy, D. S. Kim, T. Kim, T. Mansour, Degenerate poly-Bernoulli polynomials of the second kind, J. Comput. Anal. Appl., 21(5) (2016), 954-966.

[21] D. S. Kim, T. Kim, T. Mansour, J. J. Seo, Degenerate Mittag-Leffler polynomials, Appl. Math. Comput., 274 (2016), 258-266.

[22] T. Kim, D. V. Dolgy, D. S. Kim, Symmetric identities for degenerate generalized Bernoulli polynomials, J. Nonlinear Sci. Appl., 9(2) (2016), 677-683.

[23] D. S. Kim, T. Kim, Symmetric identities of higher-order degenerate q -Euler polynomials, J. Nonlinear Sci. Appl., 9(2) (2016), 443-451. 
[24] T. Kim, Symmetric identities of degenerate Bernoulli polynomials, Proc. Jangjeon Math. Soc., 18(4) (2015), 593-599.

[25] T. Kim, J. J. Seo, On the generalized degenerate Euler numbers and polynomials, Proc. Jangjeon Math. Soc., 18(4) (2015), 537-546.

[26] T. Kim, H. I. Kwon, J. J. Seo, Identities of symmetry for degenerate q-Bernoulli polynomials, Proc. Jangjeon Math. Soc., 18(4) (2015), 495-499.

[27] T. Kim, J. J. Seo, A note on partially degenerate Bernoulli numbers and polynomials, J. Math. Anal., 5(5) (2015), 1-6.

[28] T. Kim, Degenerate Euler zeta function, Russ. J. Math. Phys., 22(4) (2015), 469-472.

[29] D. S. Kim, T. Kim, D. V. Dolgy, Degenerate poly-Cauchy polynomials with a q parameter, J. Inequal. Appl., 2015:364 (2015), 15pp.

[30] T. Kim, On the degenerate Cauchy numbers and polynomials, Proc. Jangjeon Math. Soc., 18(3) (2015), 307-312.

[31] H. I. Kwon, T. Kim, J. J. Seo, A note on degenerate Changhee numbers and polynomials, Proc. Jangjeon Math. Soc., 18(3) (2015),295-305.
[32] D. V. Dolgy, D. S. Kim, T. Kim, T. Mansour, Degenerate poly-Cauchy polynomials, Appl. Math. Comput., 269 (2015),637-646.

[33] D. S. Kim, T. Kim, A note on degenerate poly-Bernoulli numbers and polynomials, Adv. Difference Equ., 2015:258 (2015),8pp.

[34] T. Kim, D. S. Kim, D. V. Dolgy, Degenerate q -Euler polynomials, Adv. Difference Equ., 2015:246 (2015),11pp.

[35] D. S. Kim, T. Kim, H. I. Kwon, T. Mansour, Degenerate poly-Bernoulli polynomials with umbral calculus viewpoint, J. Inequal. Appl., 2015:228 (2015),13pp.

[36] D. S. Kim, T. Kim, D. V. Dolgy, On q-analogs of degenerate Bernoulli polynomials, Adv. Difference Equ., 2015:194 (2015), 10pp.

[37] D. S. Kim, T. Kim, Some identities of degenerate special polynomials, Open Math., 13 (2015).

[38] D. V. Dolgy, D. S. Kim, T. Kim, T. Mansour, Barnes-type degenerate Euler polynomials, Appl. Math. Comput., 261 (2015), 388-396.

[39] D. V. Dolgy, D. S. Kim, T. Kim, T. Mansour, Barnes-type Daehee with -parameter and degenerate Euler mixed-type polynomials, J. Inequal. Appl., 2015:154 (2015),13pp. 